\title{
On the Impact of Information Technologies on Society: an Historical Perspective through the Game of Chess
}

\author{
Frédéric Prost ${ }^{1}$ \\ LIG, Université de Grenoble \\ B. P. 53, F-38041 Grenoble, France \\ Frederic.Prost@imag.fr
}

\begin{abstract}
The game of chess has always been viewed as an iconic representation of intellectual prowess. Since the very beginning of computer science, the challenge of being able to program a computer capable of playing chess and beating humans has been alive and used both as a mark to measure hardware/software progresses and as an ongoing programming challenge leading to numerous discoveries. In the early days of computer science it was a topic for specialists. But as computers were democratized, and the strength of chess engines began to increase, chess players started to appropriate to themselves these new tools. We show how these interactions between the world of chess and information technologies have been herald of broader social impacts of information technologies. The game of chess, and more broadly the world of chess (chess players, literature, computer softwares and websites dedicated to chess, etc.), turns out to be a surprisingly and particularly sharp indicator of the changes induced in our everyday life by the information technologies. Moreover, in the same way that chess is a modelization of war that captures the raw features of strategic thinking, chess world can be seen as small society making the study of the information technologies impact easier to analyze and to grasp.
\end{abstract}

\section{Chess and computer science}

Alan Turing was born when the Turk automaton was finishing its more than a century long career of illusion ${ }^{1}$. The Turk automaton was supposed to be a machine playing chess. Actually it was operated by a human hidden in it (it took many years for the hoax to be found). Last year the french chess federation suspended three titled players. They have been convicted of cheating using chess engines during the chess Olympiad that took place in Khanty-Mansiysk on September 2010. In a century tables have completely turned: nowadays it is the machine that is hidden within the player.

Computer science, and more broadly information technologies, have changed the world so deeply, so quickly and so unexpectedly that it is difficult to grasp. Economists are fond of paradoxical indexes such as the Big-Mac index [PP03] (illustrating the purchasing-power parities among currencies) or the skyscraper index [And99] (a correlation between skyscraper building and economic crises) that underly strange and funny correlations between a priori unrelated phenomenons. In this paper we develop such an index by showing how the interactions between the world of chess and computer science turn out to be particularly illuminating regarding the societal impacts of information technologies. In the same way that chess is a metaphor of war, we advocate that the interplay between information technologies and the chess world can be

\footnotetext{
${ }^{1}$ Though it can be noted that in 1912 Leonardo Torres y Quevedo built a real machine that could play King and Rook versus King endgames. It is arguably the first real chess playing machine built in history.
} 
viewed as a metaphor of the more general issue of how information technologies and society have interacted together. Moreover, as we will show, it has not been exceptional for the chess world's use of information technologies to precede mainstream uses. Thus, looking at today's relations between the world of chess and information technologies could be telling for the future of our digitalized era. Finally, the world of chess is smaller than the real society. In the same way than chess captures the essence of strategic thinking in a concise and formalized way, the world of chess can be seen as a miniature version of society making it much easier to grasp and analyze.

The game of chess has already been used as an index of the social, and geopolitical, situation of the world. In Kas03 G. Kasparov shows how the best chess masters (and style of play) of every epoch have deep links with the most prominent ideas, and geopolitical conflicts. One of the first famous chess players was Ruy Lopez, a spanish priest of the 16th century. At the time Spain was dominating the world and was conquering the "new world". Then came the Renaissance and not surprisingly one of the best players, Domenico Lorenzo Ponziani, was from Modena in Italy. The next century was the one of the philosophers of the enlightment and its blind beliefs in rationalism: the best player was of course a french, François-André Philidor, and his famous saying 'the pawns are the soul of chess' was a clear announcement of the french revolution. The great rivalry between France and Great-Britain during the 18th and 19th centuries found an echo in the fights between french and britton players: La Bourdonnais vs Mc Donnell and Saint-Amand vs Staunton to cite but a few. The parallel between the world of chess and that of ideas and geopolitical standings has continued until today (with the more than famous match Fisher vs Spassky in the middle of the cold-war). It suffices to look at the reigning world champions to see that the world has changed: Viswanathan Anand comes from India and the women world champion, Hou Yifan, is Chinese.

\section{Chess as a tool to discover computer capacities}

The game of chess has been intimately related with computer science from the early beginnings of the latter. Indeed, the founding fathers of computer science, artificial intelligence and information theory, respectively Alan Turing, Norbert Wiener and Claude Shannon proposed programs and principles for chess programs in the early fifties. A time where computers were rather product of the minds than real objects ${ }^{2}$.

At the time the questions in computer science were very fundamental and theoretical. The computer was a new artefact and it was not clear at all at what it could be used for, and where its limits were, both from a theoretical and a practical point of view. It may seem paradoxical since a computer is a very elaborated machine, which has not been invented or found by serendipity. Nonetheless, once created its scope remained largely unknown. So one could wonder: how come someone built a very elaborated machine without precisely knowing what it would be used for? The short answer is that Turing machines were invented as a (negative) solution to the very fundamental question of the decidability of logic. The universal Turing machine was a by-product of a proof regarding a theorem about the foundations of mathematics. More precisely the question was to find a generic method to state whether any given mathematical formula is true or not, together with a proof of this. One could argue that there is nothing farther from a practical perspective than this fact.

\footnotetext{
${ }^{2}$ It has to be noted that Charles Babbage and Ada Lovelace, more than century ago, imagined that chess could be programmed using numbers to represent chess position in the analytical machine.
} 
Therefore, it is natural that the first interactions between chess and computer science were focused on the investigation of the potentialities of the computer. In this perspective, the game of chess was seen as an interesting problem in order to unveil computer capabilities. The answers of the three founding fathers were all about the possibilities of the computers examined along three different perspectives: from an algorithmical point of view, from a practical point of view and from a philosophical/fundamental point of view.

In Wie65] Wiener gives an algorithmic answer to the problem of chess programming. He exposes the raw principles of chess programming (which have not fundamentally changed until today), and shows how it is conceptually possible to program a decision algorithm by the combination of a minimax algorithm paired with an evaluation function (he considers a fixed depth search).

In Sha50 Shannon gives very telling motivations on why the game of chess is especially well suited in order to discover the possibilities of computers. Indeed he writes:

This paper is concerned with the problem of constructing a computing routine or "program" for a modern general purpose computer which will enable it to play chess. Although perhaps of no practical importance, the question is of theoretical interest, and it is hoped that a satisfactory solution of this problem will act as a wedge in attacking other problems of a similar nature and of greater significance.

It is clear for him that the quality of play, or the strength, of the computer are not the primary aims: he is looking for a problem that is not a mere computation. Indeed, he adds some very interesting remarks that strike by their premonitory status (remember that the paper was written in 1950 in an era where computers were barely existing), on why the game of chess is very interesting to study:

Machines of this general type are an extension over the ordinary use of numerical computers in several ways. First, the entities dealt with are not primarily numbers, but rather chess positions, circuits, mathematical expressions, words, etc. Second, the proper procedure involves general principles, something of the nature of judgement, and considerable trial and error, rather than a strict, unalterable computing process. Finally, the solutions of these problems are not merely right or wrong but have a continuous range of "quality" from the best down to the worst.

In Tur53 Turing goes even deeper and unfolds the question "Could one make a machine to play chess" from the bare problem of enumerating legal moves:

i) Could one make a machine which would obey the rules of chess, i.e. one which would play random legal moves, or which could tell one whether a given move is a legal one?

to deeper philosophical questions:

iv) Could one make a machine to play chess, and to improve its play, game by game, profiting from the experience?

To these we may add two further questions, unconnected with chess, which are likely to be on the tip of the reader's tongue.

v) Could one make a machine which would answer questions put to it, in such a way that it would not be possible to distinguish its answers from those of a man?

vi) Could one make a machine which would have feelings like you and I do? 
Once again we see that at the heart of Turing's concern is the study of the computer capabilities (from raw computations to deep metaphysical concerns), and that chess is used as tool to discover them.

\section{Chess as a measure of hardware and software progress}

One of the interesting features of chess is that it can be used to measure a rich intellectual performance (a game of chess includes computations, spatial visualization, memory, long-term planing etc.) in a simple and relatively unbiased way. Moreover, due to the popularity of the game one can easily find players, or even tournaments, in order to measure the strength of a machine.

Arpad Elo designed a rating system, which bears its name, based on the assumption that the chess performance of each player in each game is a normally distributed random variable (see [Elo78]). In doing so the rating of a player can be objectively computed: the basic idea is to compute the average rating of its opponents during a given period. The performance of the player is given by his/her percentage of wins during this period. If he/she has scored $50 \%$, then the performance of the player is the average of the rating of his/her opponents. A $75 \%$ of wins gives a performance 200 points above the average. The correspondance between the winning percentage and the elo points delta is taken from a gaussian curve generally flattened at $350 / 400$ points (it means that a player with a rating 400 points higher than you is supposed to beat you $100 \%$ of the times). This rating system was first adopted by the US chess federation in 1960 and is now in use in most of the world chess federations and the international chess federation as well. Roughly speaking an average club player is ranked around 1500 elo points, over 2000 elo points are national level players. International Masters are over 2400 (around 3000 players in the world), Great International Masters are over 2500 (around 1000 players). The top ten is above 2760 and the all time record is Garry Kasparov's 2851 on the July 1999 and January 2000 lists.

Luke Muehlhauser has compiled the historical elo ratings of the strongest chess engines from 1963 to 2011 in Mue11. It is remarkable that starting with a rating around 1500 elo for the early version of $\mathrm{MacHack}^{3}$ in the mid sixties towards Deep Rybka 3 and its estimated 3200 elo of 2011, the slope of progress has been strikingly linear. The progress of chess engines is hard to analyze in detail because there are so many factors to take into account, but an intuitive explanation can be the following: Ken Thompson (another founding father of computer science deeply interested in computer chess) made some very interesting experiments with Belle ${ }^{4}$, see CT82. He experimentally discovered that, on average, a single extra ply in the search depth corresponds to 200 elo points. Thus the linear rate of progression of chess engine could be seen as a corollary of the Moore's law: the exponential progression of computers matches (up to some constant) the exponential combinatorics of possible moves in a game, and finally results in a linear progression in engines chess strength.

To put this progress into perspective, from an hardware point of view, one has to remind that MacHack was running on an IBM 704 that was able to execute up to 40,000 instruction per second with a memory of $5 \mathrm{MB}$ on tapes. On the other side of the spectrum, a computer cluster has been designed for Rybka with 656 GB of RAM (and 5 TB of of solid-state disks)

\footnotetext{
${ }^{3}$ Interestingly MacHack won a game vs Hubert Dreyfus in 1967, a professor of philosophy at MIT who was hired to explore the issue of artificial intelligence. He wrote an essay, What Computers Can't Do [Dre78] in which he exposed his controversial views on artificial intelligence. He also stated at the time that "a ten year-old can beat the machine".

${ }^{4}$ Belle won every tournament and world championship from 1980 to 1983
} 
and 296 processors that can each execute up to 3.33 Giga instructions per second.

It became clear to anyone in the early sixties that computers were capable of playing decent chess, among other things. The question slightly shifted towards the speed of improvement and the limits of the computer strength: when would the computer be able to beat chess experts? The society was slowly integrating the idea of computers and, as it is the case for every major scientific breakthrough, the reaction was a subtle blend of fears and excessiv optimism. The perfect illustration of these contradictory feelings is, once again, symbolically given through chess. It takes the form of a game of chess between a computer, HAL-9000, and an astronaut in Kubrik's "2001, A Space Odyssey” movie.

\section{Chess as a pionneer of a computarized economy}

Everything changed in the eighties with the apparition of personal computers and the democratization of electronic equipment. Computers suddenly (in a decade give or take) ceased to be an affair of specialists to become everybody's affair. In 1977 Fidelity Electronics Chess challenger 1 was the first chess computer available for consumers. It was a computer dedicated to chess that looked like a chess set together with a rudimental interface to input and output the moves that looked like very much like a calculator. Here again we can see that the use of computers by the chess world announced broader social uses. Indeed, the more than famous "Speak and Spell" by Texas Instrument (remember "E.T. the extraterrestrial" ? E.T. was using a hacked "Speak and spell" to call home), often presented as a precursor of the electronic devices and toys of the eighties, was only available from 1978.

Together with the democratization of computers, the eighties marked a turning point in the use of computers. At first computers were used to ... compute things ! Indeed, in order to simulate complicated physical phenomenon like weather forecasts, solving fluid mechanics equations, computing ballistic trajectories etc. you need a lot of computational power. Yet, together with the increasing of storage capacities another important use of computers emerged: databases. Database management does not require complicated computations. It is the amount, and the structure, of information that are hard to handle by hand. It is exactly where the computer can be useful at. Once again the chess world understood very quickly the advantages of computers to handle a large amount of information. Chessbase $G m b H$ is a company that was founded in 1985: this company proposed a chess database that was soon adopted by chess experts (whereas at the time chess experts did not use the chess engines that were too weak to help them in any way). As shows the following quote of G. Kasparov from [Che11, the arrival of chess databases changed everything in the preparation of matches for professional players:

In January 1987 I was back to play another 'simul' against the Hamburg team. This time I had two days to prepare, so we dug out the names of all the players and checked their records in the computer. It was an eye-opener for me. It took about ten minutes to find 192 games. If I ask my trainers to find me a game, going through the books, it could take days. This time, armed with the information I needed, I beat six of the Hamburg team and drew with the other two. The result, 7-1, was extraordinary. They couldn't believe it. Because I knew their habits, I could lead them into traps.

This prefigures in a striking way the corporation's productivity gains thanks to computers and information digitalization. What were repetitive tasks of information administration done by hand became quicker and automatic. Moreover, it became possible to perform those tasks 
without having to deal with a third part like a secretary (the trainers looking for specific games in the quote of G. Kasparov), providing a more direct access to information to managers.

\section{Chess over the network}

Another unexpected usage of computers started in the early 1990's with the quick democratization of networks: computers became able to communicate and build communities of people sharing common interests across the world. The possibilities opened by this extra feature, at first limited to the academic world, were quickly developed to play with distant people. The Internet Chess Server opened in January 1992 and was amongst the firsts online game servers. It was not uncommon to find several hundreds of players simultaneously playing. Due to this success many clones appeared in several countries: german ICS, french ICS, dutch ICS etc. It was at a time when there was not yet a web browser (Mosaic appeared in 1993) and where the web traffic was literaly exploding.

Almost every features of today's social networks were already present, albeit in a primitive way, on those chess servers. It was possible to chat, to give a short presentation of ourselves (limited to 10 ascii lines), to define a list of friends, a list of banned people etc. There were also special communication channels regarding the subject of the topic (opening theory, technical issues, french speaking channel, etc.). At the time, in 1992, I was in the first year of study at "Ecole Normale Suprieure de Lyon". I remember that I had problems to make normal people (that is people who were not studying computer science) understand what the internet was: the easiest way for me was to explain that it allowed me to play a live game of chess with some unknown person located on the other side of the earth.

It is during this period that the information spread reached the speed of liberation. The emblematic CNN television channel is often given as the example of the fact that the world was becoming a small village in which everyone was going to know everything almost instantly. Interestingly the same phenomenon occurred in the chess world, though in a more premonitory form, notably through M. Crowther's web site : "The Week in Chess" Cro95 (often called TWIC). The web site was collecting every week, with the help of volunteers, all results and game scores of chess games played in tournament through the whole world. If CNN was a worldclass broadcast news channel, it was still based on the old paradigm of top-down approach to information. What is striking in the example of TWIC is that it announces what had later been pompously called web 2.0 : a society more horizontal in which information does not come from the authorities to the people, but where information are gathered by individuals. TWIC was quickly adopted by professional players who were feeding their databases with the latest games every week (a phenomenon which gradually announces the digital convergence of the following decades). It also marks the beginning of the end of the age of obscurity (others would say the beginning of the era of massive surveillance). Suddenly every tournament chess game became available and known instantly around the world.

Even an individual game like chess gave rise to collective intelligence emergence thanks to networks. In 1999 G. Kasparov played a game in which he challenged the whole world over the internet KK00. Actually G. Kasparov was facing a team experts that gave several moves among which internet individuals had to vote: the most voted move was played. The game had a phenomenal success and it is estimated that more than 50,000 individuals from more than 75 countries participated. From every standard the game was of a very high quality. This game was a peculiar precursor of massively open source projects (i.e. projects not limited to professionals computer experts) in which a loose collection of individuals are gathered together in order to achieve a very elaborate product. Today this is best illustrated through the success 
of Wikipedia that was launched in 2001.

\section{Digital convergence and pervasive computing}

On the front of computer chess it gradually became clear that one day computers were going to be better player than the best humans. The only remaining question was when such a step would be done. In 1968 David Levy, international master, made a bet that no program would win a chess match against him within ten years. D. Levy won his bet and continued matches versus machines, he finally lost his first match in 1989 against Deep Thought.

In 1990 A. Karpov, vice world champion at the time, lost a game in a simultaneous event against Mephisto (a descendant of chess challenger), and in 1992 G. Kasparov won a match of blitz games (five minutes for the whole game) vs Fritz 2, winning 6 games, tying one but losing 4 games. It was the first time that a computer chess program won a game vs the world champion at speed chess. The first victory of a chess computer in standard tournament conditions was the one of Deep Blue vs G. Kasparov in 1996 (though Kasparov won the match 4-2). Finally, G. Kasparov lost a match vs Deep Blue in may 1997 though the history of matches was not finished: for instance in 2002 V. Kramnik drew a match vs Deep Fritz winning and losing two games, he finally (still world champion at the time) lost a match 4-2 vs Deep Fritz in december 2006.

By the beginning of the 2000s the computers, because of their strength and constant availability, started to be used as a sparring partners by chess experts. Chess players tested opening ideas and were starting to use computers to analyze their own games (looking for tactical blunders, missed defenses etc.). It was becoming possible for everyone to have an expert at home helping them to progress. It is not unlike what happened in a lot of domains like music, picture and movie editing etc. in which a lot of what was limited to professionals became consumer grade.

But the most striking feature of the technological evolution was the slow but steady integration of different softwares and information sources together. It was very natural in the world of chess: to pair a chess database together with a chess engine (that can give you some advice on the position you are looking at) was an idea present from the start of chess databases. As we saw, TWIC was a first way to feed the database with a constant flow of information, by collecting every tournament games each week. More and more tournaments were starting to broadcast live the games: the chessboards are equipped with magnets and moves are directly transmitted through the internet to chess servers like ICS. On those servers you have hundreds of spectators, and chess engines, giving their evaluation of the position, commenting the moves. The databases started to be fed live and the computer-aided analysis of the game also became instantaneous while few years back you had to write a book and to analyze the game with the help of Masters to obtain similar results.

The convergence took a step to the next level with the conjunction of smartphones (or ultra portable computers) with pervasive internet access. It is nowadays possible to connect to huge chess databases through the internet almost everywhere and to have world class level software running on your smartphone at the same time. The unexpected result of this combination is the appearance of numerous cheating controversies in chess tournaments at every level: from the world championship, and the unglamorous so called "toiletgate" or "Bathroom controversy" of the 2006 chess world championship between V. Kramnik and V. Topalov ${ }^{5}$, to local tournaments

\footnotetext{
${ }^{5} \mathrm{~V}$. Topalov's team emited suspicions about the fact that V. Kramnik was spending long period of times in the bathrooms during the games, and that it was the only place not under video and radio surveillance. This controversy led to Kramnik forfeit in game number 5 .
} 
in which random players are seldom caught using, or suspected of having used, electronic equipment to cheat. Perhaps the more striking and elaborate case of cheating is the one mentioned in the introduction. Three titled players of the french team have been convicted of cheating during the 2010 chess olympiads. This case was remarkable in how it illustrates the new capacities of information technologies. In a nutshell the fraud was built like this: games were broadcasted live from Khanty-Mansiysk, Siberia, on the internet. In France, a master was analyzing the moves with a strong chess engine and a big chess database. Once an interesting move or variation was found he texted through a cell phone the move to a third player (actually the french team coach) who would indicate the move to be played through coded gestures. You have it all: network, databases, strong artificial intelligence, pervasive communications. In Rog11 economist K. Rogoff starting from this specific affair goes as far as seeing in this the premises of a radical shift in our economy. Consider this quotation:

As skilled labor becomes increasingly expensive relative to unskilled labor, firms and businesses have a greater incentive to find ways to "cheat" by using substitutes for high-price inputs.

What is called cheating in chess translates into productivity gains in business. K. Rogoff argue that it could be the case that a lot of decisions that are taken by humans, and previously thought to be only manageable by humans, could actually be automated.

\section{The digitalized era}

Today, the links between the world of chess and the information technologies are more intricate than ever. In an interview for Time Magazine [Har11, M. Carlsen, current number one on the chess rating list, said that he was not certain whether he has an actual chess board at his home : "I might have one somewhere. I am not sure". It gives a startling illustration of the degree of virtualization reached in our society.

One can see direct influences of the information technologies on the game of chess. Overall chess players are tougher today, they have a more pragmatic approach and better defensive skills than before. This is largely due to the resilience of chess engines in difficult positions: computers have influenced the style of play of the new generations. Indeed a large amount of children have made their first step as chess players vs chess machines. Another point to notice is that it seems that today's players have a broader chess culture: thanks to databases, it is possible to browse through thousands of games very easily. Because of this players tend to change their openings more often instead of repeating the same schemas in order to get over the opponents preparation. P. Svidler (six times champion of Russia), went as far as saying (admittedly he was half serious) that "the future belongs to 1. g3", a completely offbeat way of opening the games just to avoid any kind of preparation and play chess. This is the bright side, there is a darker one.

If the chess culture is broader it is also much lighter than before. Typically in todays tournament the average player goes to the internet to get the pairing of the next round of his tournament. Then he looks for the games of its future opponent in his database and quickly spots what are the openings played by his opponent and where are his weaknesses. Then starts the so called preparation of the game: roughly it consists in the very quick visualization of 5 to 10 model games (helped with a chess engine to find whether or not the opponent make typical mistakes) just before the round.

In the preparation process the player completely relies on the machine and its judgments. This can lead to disastrous results even at the highest levels. In the 2004 chess world match 
between V. Kramnik and P. Leko, V.Kramnik lost the 8th game without having played a single move of his own. He blindly trusted an opening preparation (partially based on computer evaluation) that appeared to be flawed. As a chess trainer for kids I can testify that the faith in the machine sayings is somewhat terrifying. Very young children can tell you that this move is better than this one because the computer says it evaluates the position as +0.26 for white in this variation instead of +0.17 . I am afraid that this blind faith in machines and lack of critical spirit will generalize in our society. An interesting question is how the tension will be resolved between this blind faith in what the machine says vs the more open mind that machines help to create. Indeed, thanks to the databases and the heartless machine's evaluation, what would have been called ugly/crazy moves or ideas are tried. From this point of view computer chess has been an eye-opener: chess player become more pragmatic and less dogmatic.

Another important evolution of chess is the ever shortening of time controls, especially regarding online chess. When I started to play chess online the basic time control was 2 minutes with 12 seconds added after each move (say 8 to 10 minutes per side for the whole game since on average a game lasts 40 moves). Nowadays it is almost impossible to find someone to play at such a slow pace. The average blitz game is 3 minutes per side. There is even a new time category (standard time controls were divided along three categories: classical chess with 2 hours for the first 40 moves, rapid chess with 20 minutes for the whole game and blitz which was traditionally 5 minutes per side for the whole game) called bullet or lightning for games with less than 2 minutes per side for the whole game. Needless to say that if it allows greater quantity, the quality of the play is severly harmed. From a thinking and meditating game chess has become a game of interactions and reflexes thanks to computers: indeed it is much easier to play quickly with a mouse than with actual pieces and clocks. The field of human-computer interactions has also its word to say in this race: many chess server interfaces have the "premove" feature. That is to allow the player to actually program his move even before his opponent has made its own move. This is another warning for our digitalized society: to move in advance without having actually waited for the move of the opponent is a wonderful metaphor of a twitter driven society.

\section{References}

[And99] L. Andrew. The skyscraper index: Faulty towers. Technical report, Property Report. Dresdner Kleinwort Waserstein Research, 1999.

[Che11] Chessbase. Chessbase is 25. web: http://www. chessbase.com/newsdetail. asp?newsid=7229, 2011.

[Cro95] M. Crowther. The week in chess. web: http://www.chess.co.uk/twic/, 1995.

[CT82] J.H. Condon and K. Thompson. Belle chess hardware. In M.R.B. Clarke, editor, Advances in Computer Chess 3. Pergamon Press, 1982.

[Dre78] H. Dreyfus. What Computer can't Do. Harpercollins, 1978. Revised edition.

[Elo78] A. Elo. The Rating of Chess Players, Past $\&$ Present. Ishi Press, 1978.

[Har11] E. Harrel. A bold opening for chess player magnus carlsen. Time Magazine, January 112011. availabel at: http://www.time.com/time/magazine/article/0, 9171, 1950939, 00.html.

[Kas03] G. Kasparov. My Great Predecessors - Part 1. Everyman publishers, 2003.

[KK00] G. Kasparov and D. King. Kasparov Against the World: The Story of the Greatest Online Challenge. KasparovChess Online, 2000.

[Mue11] L. Muehlhauser. Historical chess engines estimated elo ratings. web: http://lukeprog.com/ special/chess.pdf, 2011. 
[PP03] M. R. Pakko and P. S. Pollard. Burgernomics: A big mac guide to purchasing power parity. Technical report, Federal Reserve Bank of St. Louis, 2003.

[Rog11] K. Rogoff. Technology and inequality. web: http://www.project-syndicate.org/ commentary/rogoff82/English, 2011.

[Sha50] C. Shannon. Programming a computer for playing chess. Philosophical Magazine, 41(314), March 1950.

[Tur53] A. Turing. Faster than thought, chapter Digital Computer applied to games. Pitman Publishing, 1953. Available at turingarchive.org: http://www.turingarchive.org/browse.php/B/7

[Wie65] N. Wiener. Cybernetics, Second Edition: or the Control and Communication in the Animal and the Machine. The MIT Press, 1948 (second edition 1965). Second Edition. 